\title{
TORSIONAL RESPONSE OF FIXED BASE CONNECTION UNDER COMBINED CYCLIC BENDING-TORSIONAL LOADING
}

\author{
Mohamed A. Mohamed, Sherif S. Safar and El-Sayed B. Machaly
}

Faculty of Engineering, Cairo University, Cairo, Egypt

\begin{abstract}
ملخص البحث

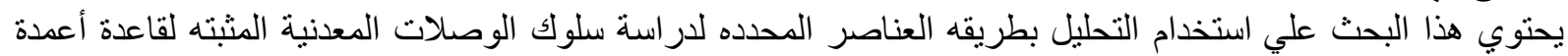

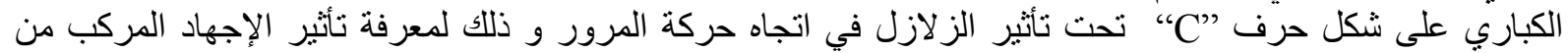

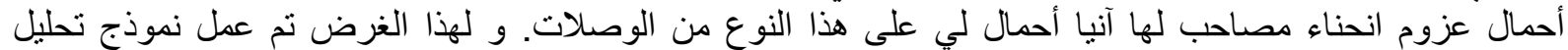

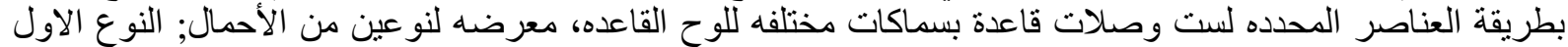

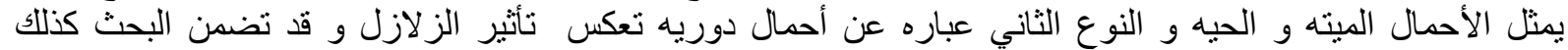

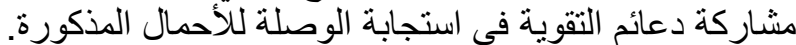

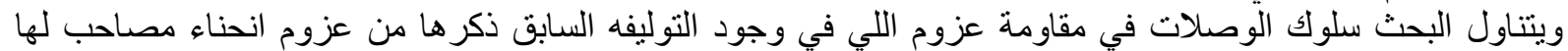

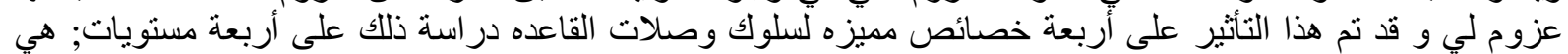

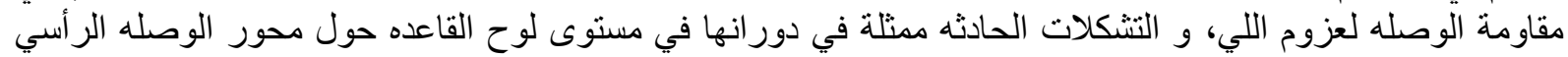

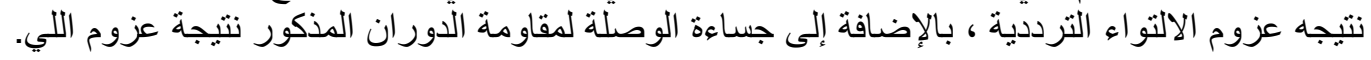

\begin{abstract}
There is a lack of study concerning the torsional effect on the torsional response of column base especially cyclic behavior under combined torsional-flexural loading; many unknowns exist on this topic. A study for the torsional performance of the base connection for C-bent column subjected to seismic in the traffic direction is necessary to address the effect of the complex combination of cyclic flexural-torsional action on such a type of connection; for this purpose an extensive numerical parametric study was conducted to evaluate the torsional performance of the fixed base connection of C-bent bridge pier against combined cyclic bending and torsional loading following SAC phase II protocol. After verifying results with previous experimental works a Finite Element Analysis (FEA) using Strand7 is performed for Finite Element Models (FEM) which simulates force transfer at major contact interfaces in the connection, and a total of six base plate connection sub-assemblages with variable base plate thicknesses (three for stiffened connection and three for un-stiffened connection) anchored by 10 bolts, were configured and analyzed to support double cantilever pier subjected to eleven loading schemes that reflect different pairs of gravity moment and axial loads along with cyclic loading in the traffic direction eccentric from pier center of rigidity to guarantee the application of combined flexural-torsional action on the base connection. The study provided a curves for the following performance terms; strength (ultimate torsion), deformation (ultimate twisting angle), and stiffness (torsional stiffness); the seismic torsional performance of the base connection under the previously mentioned loading package was evaluated by plotting the variation of the mentioned terms against the parametric values of the base plate thickness for different loading condition considered in the analysis.
\end{abstract}

Keywords: C-Bent Pier, SAC Protocol, Rotation-Drift Ratio, Torsion, Gravity Loads Eccentricity, Rotational Stiffness, Curvature. 


\section{INTRODUCTION}

Column base connections are critical load path elements, transferring loads to the foundation and transmitting ground accelerations due to earthquakes to the superstructure.

Figure 1 is a statistical analysis of over 600 steel frame buildings, compiled after the 1995 Hyogo-ken Nanbu earthquake, buildings were classified into three categories: 1) buildings built to the previous code (before the 1980 Building Standard), 2) buildings built to undefined code provisions, and 3) buildings built to the 1995 provisions. The comparison shows that column base damage was the most common form of damage in all three building categories [1].

Because of the urban areas space limitation, bridges with particular configurations, such as C-bent column bridges, skewed bridges and curved bridges, are often used. Due to their irregular structural configurations, the special seismic consideration is required to design these bridges. Since superstructure mass is eccentric from center of rigidity in C-bent columns (scope of this paper) as shown in Figure 2, seismic forces in the traffic direction causing forces out of plan of $\mathrm{C}$-bent column that induce a combination of seismic torsion and other internal forces that would result in the complex flexural and shear failure of base connection for these bridges [2].

Many researches handled the cyclic flexural behavior of the fixed base connection however there is a lack of study concerning the torsional effect on the column base especially cyclic behavior under combined torsional-flexural loading; many unknowns exist on this topic.

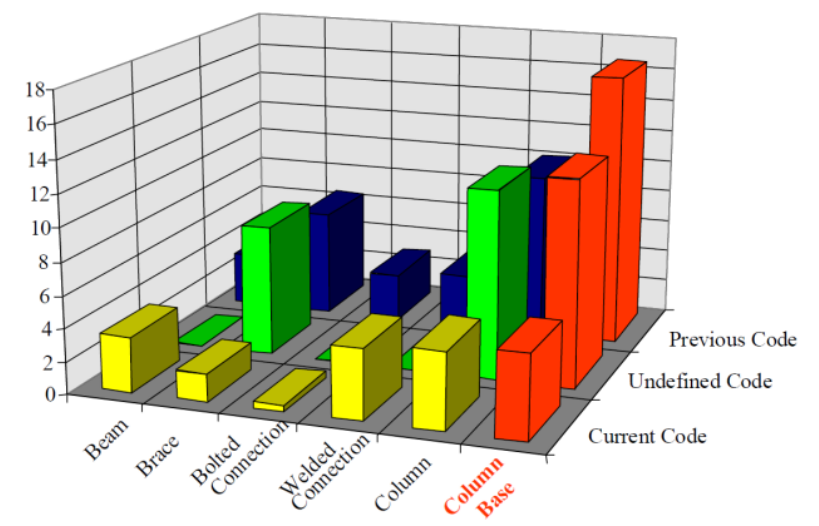

Figure 1: Statistical analysis of the structural damage compiled after the 1995 Hyogo-ken Nanbu earthquake [1].

Because of the importance of base connection and complex combination of forces mobilized on base of C-bent columns due to seismic actions; a study for the performance of the base connection for C-bent column subjected to seismic in the traffic direction is necessary to address the effect of a combined cyclic flexural-torsional action on such a type of the connection.

Many researches that studied the cyclic behavior of base connection has utilized cyclic loading scheme that yields to SAC phase II loading protocol for stepwise increasing cyclic test shown in Figure 4 that controlled by interstory drift angle which is defined by the interstory displacement divided by story height [3].

Drift capacity of strong column weak connection was investigated through a series of experiments that investigated the nonlinear, cyclic behavior of column base connections found that strong column, rigid base plate/weak anchor connections were able to accommodate drift ratios greater than 0.04 [4]. Also, at the end of the tests nonlinear rotation at the base caused for permanent deformation at the top of the column that is so hard to be straightened [5].

Many sources of deformation would contribute in the story drift of the column attached to fixed bas connection, as investigated for exposed column bases in buckling restrained braced (BRB) frames significant slip of the base plate was observed when the BRB force changed from compression to tension. In the design, not only the shear resistance should be checked, but also the deformations at serviceability and ultimate limit state[6]. Further experimental work has been conducted showed that the contribution of shear deformation to 
the overall displacement is relatively small. The fixed-end rotation of the plate and the flexural deformation of the column account for a large portion of the tip deflection in all tested connections [1].

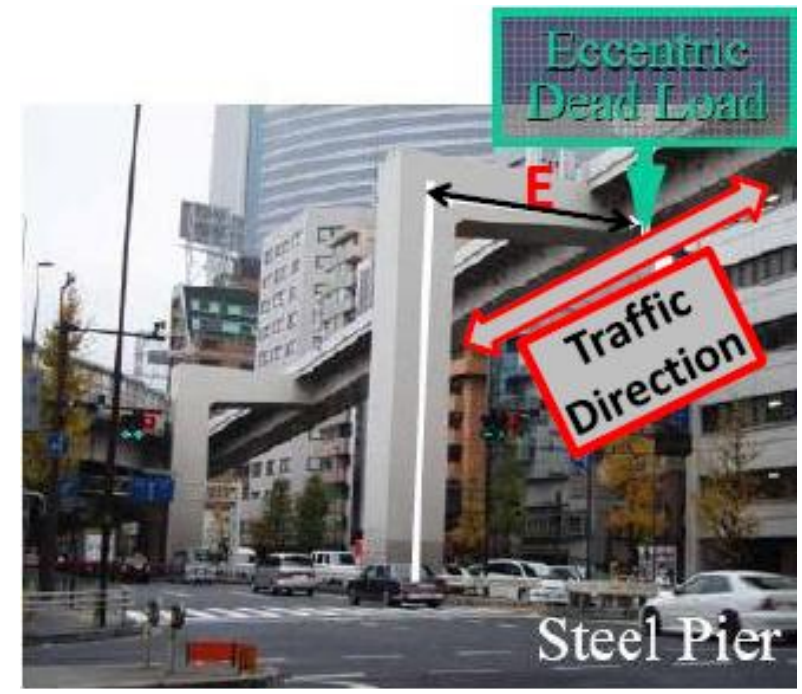

Figure 2: Steel C-Bent Bridge Pier

From the other hand modelling technique of base connection has a significant impact on the displacement response for the numerical analysis of frame which was investigated for cablestayed bridge tower as reported in [7]; at the early time of time history response before the formation of hysteretic damping through the inelastic deformation of base connection components, the displacement peak response significantly decreases due to connection flexibility compared to fully rigid of fixed base model, while the acceleration response slightly changes with overridden acceleration spike [7].

Grade, stiffeness and detailing of anchor bolts has its impact on the base connection behavior and mobilized deformation weather in the column, or in the base plate deformed profile.

There was significant variation in the ultimate deformation capacity of the connections depending on both the anchor material used and the stretch length provided. In the level of anchor bolt elongation; increasing stretch length was found to increase the ultimate deformation capacity; however the increase was not linearly proportional to the increase in stretch length. In contrast, increases in material ductility (as measured by tensile tests) were found to lead to proportional increases in connection deformation capacity [4], This fact is emphasized as found in [5]; were the anchor bolt Grade 8.8 is not a reliable member to be designed weaker than any other base elements due to the brittle failure of high strength bolt. In order to prevent the fracture of the anchor bolts, they should be designed as strongest link of the base plate connections. If not, ductile anchor bolts should be used to prevent any brittle failure of them [5].

However in lateral deformation of bolt as recorded by [6] for exposed column bases in buckling restrained braced $(\mathrm{BRB})$ frames, the anchor rods experienced large lateral deformation due to the tensile and shear force on the base plate. The interaction of shear and axial stress of the anchor rods is suggested to be considered to evaluate the maximum moment resistance of the exposed column bases in BRB frames[6].

Further study was conducted on the effect of bolt arrangement and experiments revealed that the arrangement of anchor rods controlled the maximum moment resistance of the exposed column bases in BRB frames. The slip of base plate was slightly affected by the arrangement of anchor rods [6]. In the same context a numerical analysis considered the number of bolts in exposed base plate bending about weak axis of column, this study concluded that the 4-bolt connections may cause a convex-shaped out-of-plane base plate deformation, which is resulting in very complicated base plate yielding pattern on the tension side and undesirable bearing stress concentration at the corner of the grout on the compression side. For these reasons, in this study, use of 6 anchor bolts is recommended as a minimum 
number of anchor bolts for the exposed-type column-base plate connections especially in high seismic zones [8].

Also effect of anchor post tensioning was introduced in the literature. Post tensioning of the anchor bolts can highly change the seismic performance of the column base plate connection by considerable increase of rotational stiffness and ductility [5]. However as found by numerical analysis for cable stayed tower considering the base plate flexibility, the bolt pre-tension force quickly vanishes through the first few cycles within the first five seconds of the time history response [7].

Many researches verified the role of the base thickness on the base connection behavior. The variation of base plate thickness has a significant effect on the strength, ductility, and stiffness of the connections. The increase of plate thickness leads to an increase in strength and rotational stiffness of the connection. An increase of plate thickness beyond that required for forming a plastic hinge in the column is inefficient since it does not change the strength or ductility of the connection [1].

Also effect of base plate thickness is extended to the contact stress distribution, where a thinner base plate can cause high local stress concentration in the anchor bolts on the tension side due to outward base plate deformation. Hence, for a selected anchor bolt size, minimum thickness of the base plate should be provided to prevent such condition in the anchor bolts before the connection reaches its ultimate state. In order to prevent undesirable early grout crushing under the base plate edge on the compression side, designing an excessively thick base plate coupled with weaker anchor bolts should be avoided [8].

One of the most important criteria of the base plate connection is its rotational stiffness which impacts the overall behaviour of the structure under seismic event as mentioned later by [7]. The rotational stiffness of the base connection suffered a considerable drop at the end of cyclic loading. So, they show higher flexibility that can increase demands of the structures in any further earthquake [5]. However for test specimens of BRB base plate connection, the moment-rotation hysteresis curve of the column base is pinched but not symmetry as that of the exposed column base in moment resisting frames [6]. From design standards aspect, column base connections designed according to US practice yield an initial rotational stiffness of $2.0 E I_{C} / L_{c}$ regardless of the number of rods used. The value of $2.0 E I_{d} / L_{c}$ falls between the rigid and the pinned response limits which are defined in Eurocode 3 as 25 $E I_{c} / L_{c}$ and $0.5 E I_{C} / L_{c}$, respectively [1].

many design procedures have proposed a scheme for the contact stress distribution beneath base plate however they needs further experimental / numerical verification. As performed in [7] when the base plate model considered connection flexibility rather than rigid model; the base lift-off is significantly large compared to concrete contact deformation; the distribution of compressive contact stresses under the base plate violates the uniformly distributed approximation [7].

Drake and Elkin (D\&E) proposed that a uniform distribution of the resultant compressive bearing stress is more appropriate when utilizing LRFD[9]. In the D\&E method, location of the resultant bearing force $\left(R_{u}\right)$ is highly dependent on the assumed value of compressive strength of concrete or grout beneath. However, results of the numerical analysis by [8] showed the opposite. The numerical parametric study revealed that location of the resultant bearing force $\left(R_{u}\right)$ as well as its amount varies with the change of the base plate thickness and anchor bolt stiffness. Thinner base plates and stiffer anchor bolts increase the amount of $R_{u}$ due to the shortened overall moment arm between the total tensile bolt force $\left(T_{u}\right)$ and $\mathrm{R}_{\mathrm{u}}[8]$.

Cyclic response of base plate connection is also affected by applied actions; the level of axial force has a significant effect on the strength, ductility, and failure mode of the column base connection. Reduction of axial load beyond the design level causes an increase in the ductility and a decrease in the connection capacity [1]. Even the statistical system would impose a combination of forces that affect the base behavior as reported by [6]; the horizontal component of the BRB axial force increases the shear resistance demand of the base plate of exposed column bases. And the vertical component of the BRB axial force changes the axial force on the exposed column bases significantly [6].

Combination of applied force axial, moment, and shear can control the failure mode especially in the seismic events where the mentioned combination is variable along the duration of seismic event. It was found that the worst case for column base connection design 
would be when $\mathrm{P}=0$ based on the observation that the generated $\mathrm{P}-\mathrm{M}-\mathrm{V}$ interaction was symmetric about $\mathrm{P}=0$ plane. During earthquake generated shaking, the column might go through the load level $\mathrm{P}=0$. The authors noted that, in such cases, the column base connections should be designed for the moment and shear capacity of the column corresponding to $\mathrm{P}=0[10]$. However the dead weights have slight effects on bolt pre-tension force release [7].

\section{Objective of the study}

When C-Bent bridge as shown in Figure 2 subjected to seismic action aligned with traffic direction, the C-Bent column and consequently its base connection will be subjected to two sets of forces in two perpendicular directions, first set is a permanent combination of axial and bending moments due to eccentric loading of gravity loading causing flexural action in the pier plan, this direction will be referred as transverse direction, and the ratio between bending and axial permanent Loads is depending on the eccentricity " $E$ " value illustrated in Figure 2. The other set of forces is a combined cyclic flexural-torsional stresses accompanied with cyclic shear due to seismic induced forces at center of mass eccentric from the pier center of rigidity in the direction of traffic (out of pier plan), this direction will be referred as longitudinal direction and again ratio of flexural to torsional stress is following the variation of "E".

In order to simulate the above mentioned state of stresses to capture the real behaviour; a numerical study using finite element analysis shall be conducted to study varieties of connection configuration with variable base plate thickness subjected to the previously mentioned two sets of forces with parametric values of "E" that produces different transverse bending to axial ratios combined with cyclic loading in the longitudinal direction with multiple levels of combined flexural-torsional ratios. This to address the torsional behaviour of fixed base connection supporting C-Bent columns, and how the torsional strength and twisting deformation at ultimate state are affected by the connection configuration and loading combination

Research within this paper concerns the connection behaviour, hence strong column weak connection philosophy is followed to ensure that failure will happen in the connection and the column will remain elastic.

\section{Verification Model}

\section{Selection of Model}

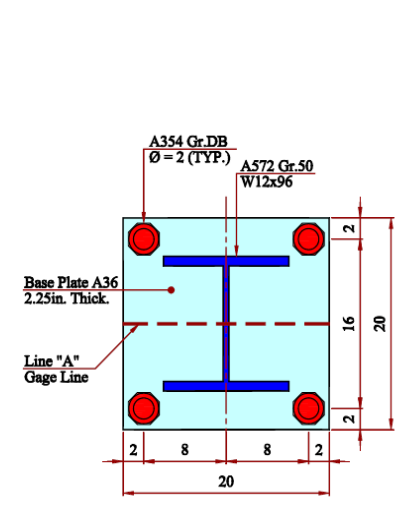

PLAN VIEW

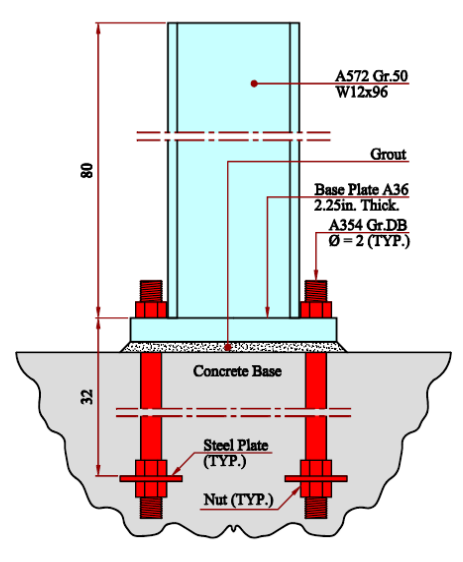

ELEVATION VIEW

Figure 3: Details of verification model (All Dim. in inch)

Table 1 Mechanical Properties of Connection Elements

\begin{tabular}{|l|c|c|c|c|}
\hline Component & Steel Grade & $\begin{array}{c}\text { Yield Strength } \\
(\mathrm{MPa})\end{array}$ & $\begin{array}{c}\text { Young's Modulus } \\
(\mathrm{MPa})\end{array}$ & Poisson's Ratio \\
\hline Anchor Bolt & A354 Gr.DB & 897 & 200000 & 0.3 \\
\hline Column & A572 Gr.50 & 344 & 200000 & 0.3 \\
\hline Base Plate & A36 & 248 & 200000 & 0.3
\end{tabular}


The verification model has been selected from international journal of steel structures (ijoss) technical paper entitled "Exposed Column-Base Plate Connections Bending About Weak Axis:II Experimental Study" [11]. The specimen is an exposed-type column-base plate connection sub-assemblage 4-bolts connection as shown in Figure 3 were tested under the SAC phase II loading protocol for stepwise increasing cyclic test shown in Figure 4 in the direction of column weak axis. The experimental study investigates the global cyclic performance of specimen and behavior of major connection elements under large column lateral displacement. Table 1 provides material properties of each components of the test specimen.

\section{Loading Scheme}

The SAC Phase II loading history is applied to ensure the results can be compared with numerous beam-to-column moment connection test results conducted during the SAC investigations. The drift in this loading history is defined as the ratio between the column lateral displacement and its effective height [3].

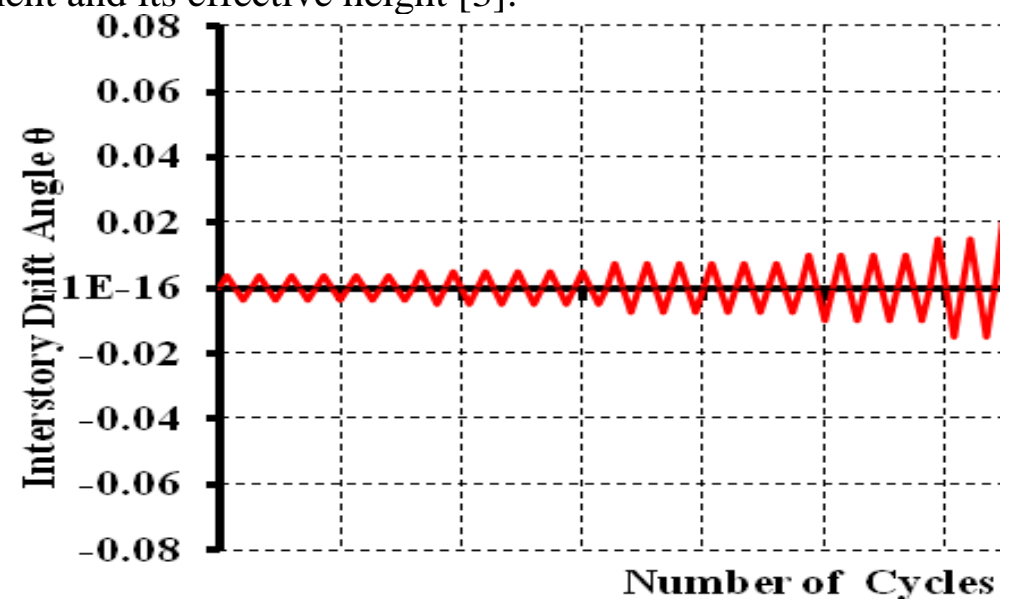

Figure 4: SAC phase II loading protocol for stepwise increasing cyclic test[3]

\section{Finite element modeling}

For the purpose of the finite element simulation finite element software Strand7 has been used. Strand7 is general purpose finite element analysis system developed by G+D Computing. Four types of elements were adopted from strand7 library to build the verification model which they are: Brick Elements, Plate Element, Beam Element, and Link Element.

Brick elements have only three translational degrees of freedom at each node, as per Figure 5 and the companion descriptive Table 3, brick element was used to model bolt, and base plate, while for the purpose of modelling column flanges, and column web a Plate/shell element was used. The plate/shell element is the most general type of plate element in that it is a three-dimensional membrane and bending element.

One of the most difficult aspects in numerical analysis for column-base plate connections is to model the three major contact interfaces, including the interfaces between (1) the nut and base plate upper surface; (2) the base plate lower surface and the grout; and (3) the base plate soffit and concrete foundation.

Figure 5 and the companion descriptive Table 3 provides finite element modelling for each of the mentioned interface. In order to model force transfer in the above three major contact interfaces, a point contact beam element was adopted such that gap opening, compression only stiffness and friction are achieved, Table 2 describes the work mechanism of each type for point contact beam element found in Strand 7 library.

Finally At grout top surface level where the bolt shear planes exist the XYZ rigid link has been assigned between fixed nodes at bolt center and the rest of nodes (which set free) at the mentioned plane as shown in Figure 5. The Rigid Link provides an infinitely stiff connection between two nodes. 
Table 2 Point Contact elements structural properties

\begin{tabular}{|l|c|c|c|l|}
\hline & Compression & Tensile & Friction & Active case \\
\hline Zero Gap & Yes & No & Yes & At gap closing \\
\hline Normal & Yes & No & Yes & In compression \\
\hline
\end{tabular}

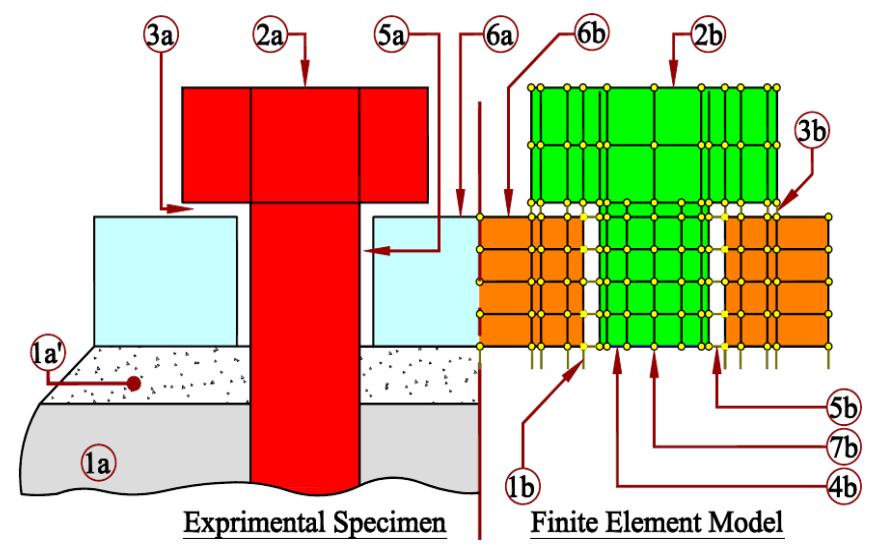

Figure 5: Elements Types for finite element modelling

Table 3 Mechanical Properties of Connection assembly components

\begin{tabular}{|c|c|c|}
\hline ID. & $\begin{array}{c}\text { Experimental Specimen } \\
\text { (a) }\end{array}$ & $\frac{\text { Finite element model }}{(\mathrm{b})}$ \\
\hline 1 & Concrete Base & Normal Contact \\
\hline 1 & Grout & Normal Contact \\
\hline 2 & Bolt & Brick Element \\
\hline 3 & Bolt-Base plate interface & Normal Contact \\
\hline 4 & ----- & Rigid link element \\
\hline 5 & Bolt-Bolt Hole interface & Zero gap contact \\
\hline 6 & Base Plate & Brick Element \\
\hline 7 & ------ & Fixed central point \\
\hline
\end{tabular}

\section{Material modeling}

In this numerical study, constitutive relationships of the steel members are simplified by elastic-perfectly plastic bilinear lines as shown in Figure 6, while concrete nonlinearity is not considered and it has been modelled as mentioned previously as a normal contact element.

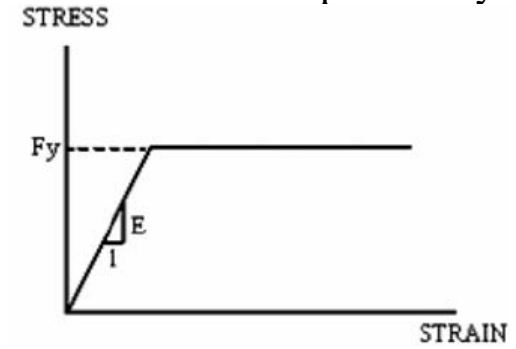

Figure 6: Constitutive relationship for steel

\section{Analysis results verification}

With considering all parameters mentioned in the above sections, the analytical results were extracted to be compared with experimental results as per figures Figure 7, and Figure 8. By viewing the mentioned figures it is found that analytical results have good agreement with experimental works. 


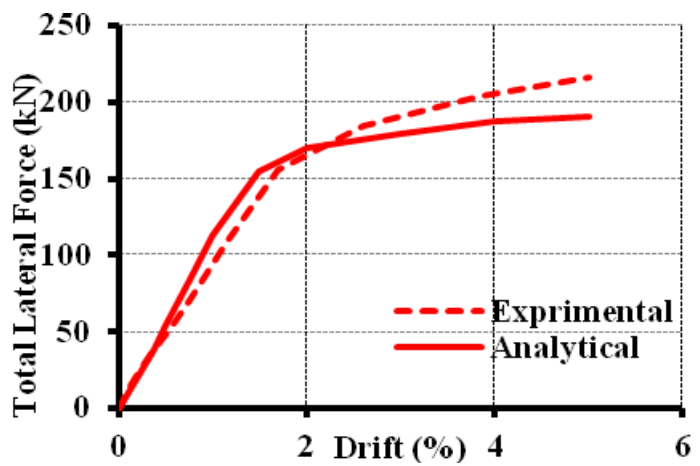

Figure 7: Comparison Between analytical and experimental results for Lateral Force

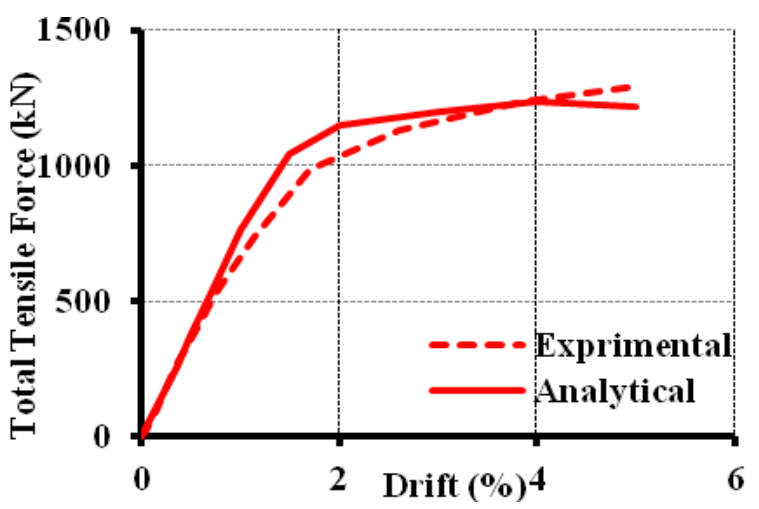

Figure 8: Comparison Between analytical and experimental results for Tensile Force

\section{Parametric Study}

Same simulation procedures, modeling techniques / element types, and Material properties mentioned in section 0 shall be followed to build up the analysis models conducted in the parametric study. The following subsections are a detailed demonstration for the factors considered in the numerical parametric study to achieve research objective. Parameters related to loading are inspired from [2] which handled the same topic, however for RC bridge pier.

\section{Analysis Model Description}

Figure 10 is an isometric detailed view for the analysis model utilized in the parametric study, Figure 10a is a global perspective for the analysis model showing that the model is a box section of center line dimension $900 \mathrm{~mm} \times 900 \mathrm{~mm}$ and $25 \mathrm{~mm}$ wall thickness forming a double cantilevered pier, with effective height of $7250 \mathrm{~mm}$ measured from the base connection to the C.G of box section, and $1350 \mathrm{~mm}$ length for each cantilever measured from the face of column, and at the end of each cantilever a cyclic loading in the longitudinal direction is applied; this load yields to SAC phase II shown in Figure 4 with a multipliers as per Table 5 dedicated for each end of the double cantilever column to count for the parametric ratios considered in the research between flexural and torsional seismic action. Also permanent loading conditions are accounted by concentrated axial load and transverse moment that their values are set as per Table 4. While Figure $10 \mathrm{~b}$ is a zoom view for the base connection of stiffened condition showing a full dimension for each component of the connection assembly, similar presentation is introduced for un-stiffened condition as per Figure 10c.

Stiffener thickness utilized is as the same as box section wall thickness $(25 \mathrm{~mm})$; connection is anchored by 10 anchors rods Dia. $65 \mathrm{~mm}$ with pattern shown in Figure 10b, and Figure 10c, the anchors are arranged such that 5 anchors are placed at the tension side of the permanent transverse applied moment and these bolts shall be entitled as tension side bolts, while the remaining 5 anchors are placed in the opposite side where permanent transverse moment imposes compressive strains and theses bolt shall be referred as compression side bolts. At last base plate dimension is $1230 \mathrm{~mm} \times 1230 \mathrm{~mm}$ with thickness subject to parametric values considered in the analysis. 


\section{Base Plate Thickness}

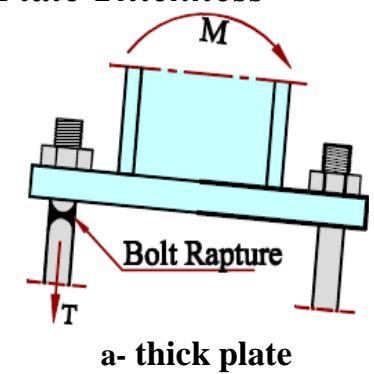

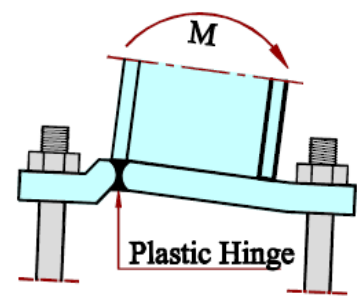

b- intermediate plate

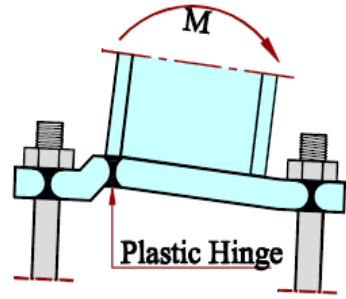

c- thin plate

Figure 9: Base plate behavior models

As per literature Behavior of base connection is sensitive to the base plate thickness. As illustrated in Figure 9 the Behavior of base plate is classified for 3 classes according to the expected mode of failure; thick plate behavior, intermediate plate behavior, and thin plate behavior. In this study three parametric values has been adopted to capture as much as possible the expected modes of failure previously mentioned, the selected values shall be $55 \mathrm{~mm}, 75 \mathrm{~mm}$, and $95 \mathrm{~mm}$.

\section{Stiffening condition}

As the vertical stiffener is an essential component in the connection, effect of stiffening condition has been assessed on two levels. First is un-stiffened base connection; Figure 10c, and, the second level is stiffened; Figure 10b. This will address the effect and contribution of stiffening condition on the connection behavior.

\section{Plastic Moment Reserve}

Permanent loads that represents the gravity actions on the base connection are decided to be represented as a ratio from the plastic moment capacity of the column section, several trial has been made to address the appropriate ratio for this purpose, and it was found that giving gravity loading (concentric or eccentric) a cut of $30 \%$ as a consumed share from the plastic moment capacity of the column, will have a noticeable presence on the cyclic behavior of the connection assembly and in the main time does not lead to unintended failure due to over stress from gravity loading which does not serve the research scope, thus all studied connections assembly shall be analyzed against initial state of stress where $30 \%$ of the column plastic capacity is dedicated to the applied permanent load, while the remaining $70 \%$ of plastic capacity is accounted as reserve strength in the column section.

Table 4 illustrates the eccentricity "E" of the applied permanent loads, which stated as a relative value of column section dimension " $D$ " shown in Figure 10. Considering what stated in 0 a broad spectrum of axial and flexural loads combination has been addressed such that $30 \%$ of the column plastic moment is occupied by the applied permanent loads that represent gravity load in the real case.

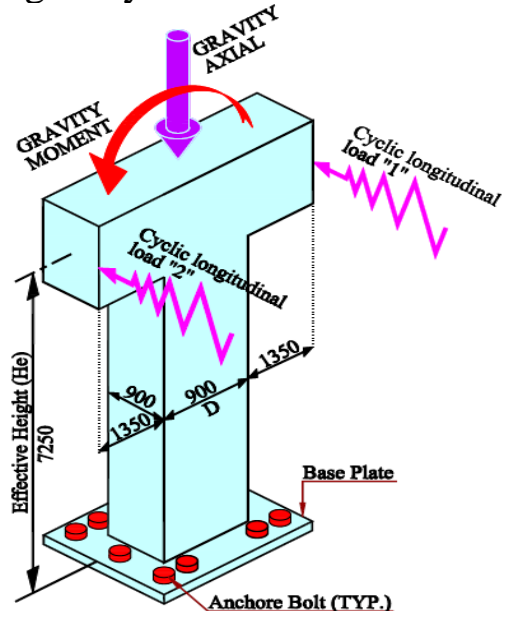

a- Perspective view of the analysis model

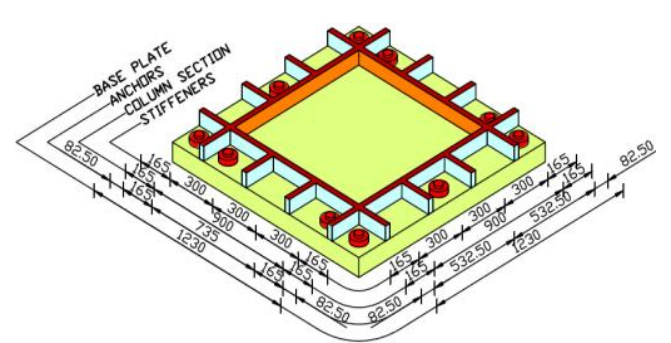

b- Detailed view of stiffened connection

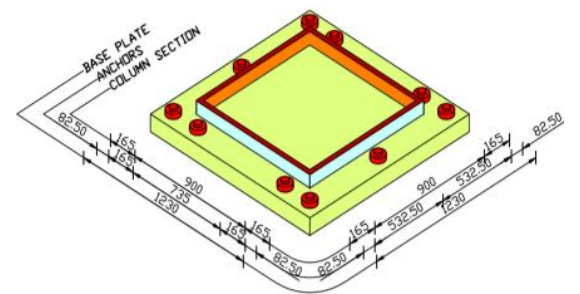

c- Detailed view of un-stiffened connection

Figure 10: Isometric View for the analysis model 


\section{Gravity load eccentricity}

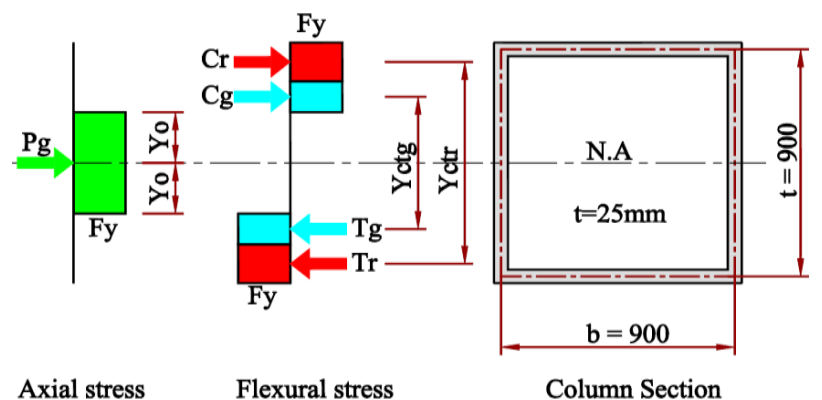

Figure 11: Plastic stresses distribution on column box section

Figure 11 is a first principle calculation for the plastic moment reserve as a couple forces "Tr", and "Cr" with lever arm "Yctr", while the remaining of stress diagram shall be dedicated to sustain combined axial load "Pg" and gravity moment couple "Tg", and "Cg" as applied permanent loads.

Table 4 Gravity loads eccentricity values

\begin{tabular}{|c|c|c|c|c|}
\hline $\begin{array}{c}\mathrm{E} \\
(\mathrm{mm})\end{array}$ & $\begin{array}{c}\text { Yo } \\
(\mathrm{mm})\end{array}$ & $\mathrm{E} / \mathrm{D}$ & $\begin{array}{c}\mathrm{Mg} \\
(\mathrm{kNm})\end{array}$ & $\begin{array}{c}\mathrm{Pg} \\
(\mathrm{kN})\end{array}$ \\
\hline 0 & 437.5 & 0 & 0 & 15050 \\
\hline 450 & 178 & 0.50 & 2750 & 6110 \\
\hline 1350 & 69 & 1.50 & 3210 & 2378 \\
\hline
\end{tabular}

\section{Rotation-Drift ratio " $r$ "}

A non-dimensional parameter called "rotation-drift ratio", $r$, is introduced here to define the level of combined cyclic bending and torsion as:

$r=\frac{\theta}{\Delta}$

Table 5 Parametric values of "r" and relevant SAC load multipliers

\begin{tabular}{|c|c|c|c|}
\hline$r$ & Load "1" & Load "2" & Remarks \\
\hline 0 & 1 & 1 & Only for reference case of pure torsion; Figure 14 \\
\hline 1 & 1.25 & 0.75 & \\
\hline 3 & 1.75 & 0.25 & \\
\hline 5 & 2.25 & -0.25 & \\
\hline
\end{tabular}

A schematic representation for the analysis model is introduced in Figure 12 to simplify the physical meaning of " $r$ " factor and its relation to the analysis model deformation. As per Figure 12 " $\theta$ " is the column rotation and " $\Delta$ " is the interstory drift angle which is defined by the interstory displacement " $\delta$ " divided by story height "He". $\Delta$ is represented in $\%$ as per Figure 4, while $\theta$ shall follow the parametric values of " $r$ " found in Table 5 below.

Further in Figure 12 Cyclic load " 1 " and cyclic load "2" shall be applied with the same frequency of loading scheme provided in Figure 4 and by the aid of the multipliers listed in Table 5 a combined cyclic torsion and bending shall be imposed such that specified parametric values of the corresponding " $r$ " are achieved. 


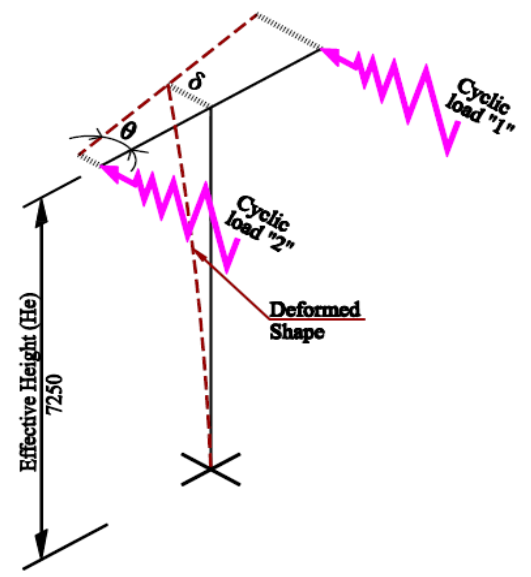

Figure 12: Schematic representation for deformed shape of the analysis model

\section{Results and Discussion \\ General}

An analytical study was performed to investigate the cyclic behavior of different configuration of fixed base connection to address the effect of combined cyclic bending and torsional loading on the connection behavior in the presence of different combination of permanent axial and transverse moment.

Results for the analytical study were categorized into the following factors: (1) strength capacity ultimate torsion $\left(T_{U}\right)$ moment; (2) deformations that determined by Ultimate twisting angle $\left(\theta_{U}\right)$; and (3) torsional stiffness for twisting actions $\left(K_{t}\right)$.

\section{Reference Values}

In order to make sense from the performed analytical study; author preferred to present results as a relative values with respect to corresponding reference amounts, this to expand the research out comings to a broader front rather than being limited to a specific cases considered in this paper, reference values shall be denoted by " 0 ", and there are two types of them illustrated in the next paragraphs.

First type of the reference amounts are extracted from an analysis results of reference connection assembly subjected to specific load cases; the author decided to set one of the already analyzed connection as reference connection assembly, which is base plate of minimum parametric thickness $(55 \mathrm{~mm})$ with un-stiffened configuration; this facilitate tracing connection behavior variation when changing base plate thickness or changing stiffening condition of the assembly. The relevant specified load for the mentioned reference connection is a pure cyclic torsional loading in the absence of permanent loads Figure 14, and the consequent reference amounts are reference ultimate torsion $\left(T_{U O}\right)$, and reference ultimate twisting angle $\left(\theta_{U 0}\right)$; neutralizing parametric loading conditions, is helpful in reading their effect on the connection cyclic response.

The second type of reference values is related to the torsional stiffness of cantilevered beam subjected to concentrated twisting moment at the cantilever end $\left(K_{T 0}\right)$, the beam shall be a perfect match of column introduced in the study (Material, section properties, and Geometry). 


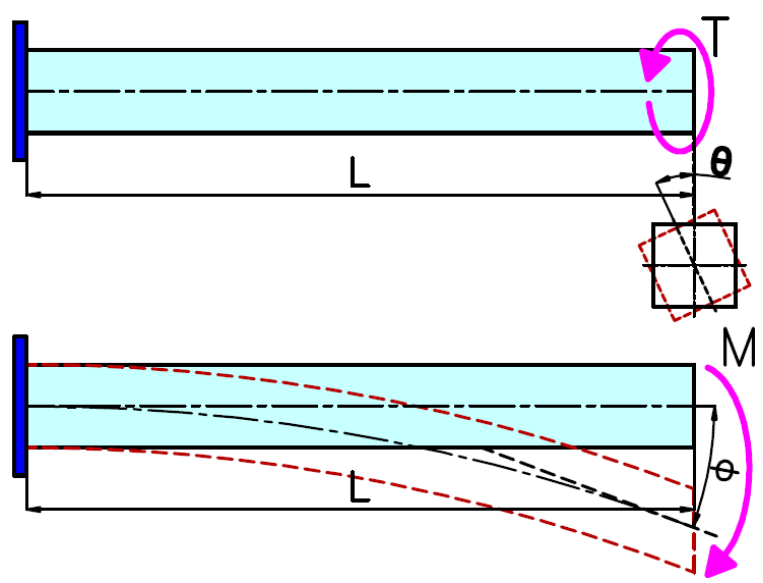

Fig. 13: Beam deformed shape under bending and twisting loading

As it well know in the mechanics of the structures that cantilever stiffness for rotation $\Phi$ at its tip Figure 14 equals to $E_{C} I_{C} / L_{C}$, where $E c, I c, L c$ are the modulus of elasticity, moment of inertia, and length of fixed cantilever respectively, also as stated in [12] that base connection shall be considered flexuraly rigid when achieving at least a value of $25 E_{C} I_{C} / L_{C}$, which is twenty five times the provided stiffness at the cantilever tip; by analogy for twisting action, if the theoretical torsional stiffness for twisting angle $\theta$ at cantilever tip is $G_{C} J_{C} / L_{C}$, hence the base connection can be considered torsionaly rigid when achieving $25 G_{C} J_{C} / L_{C}$, where $G c, J c$, $L c$ are the shear modulus, torsion constant, and length of fixed cantilever respectively. Further [12] also provided a lower bound for the column base flexural stiffness to define the pinned behavior, and the mentioned bound is $0.50 E_{C} I_{C} / L_{C}$; following the same analogy adopted for rigid model, then lower bound of base connection torsional stiffness yields to $0.50 G_{C} J_{C} / L_{C}$, which defines the pinned behavior.

Accordingly $\left(K_{T 0}\right)$ shall be set as $25 G_{C} J_{C} L_{C}$, and the concluded lower limit $0.50 G_{C} J_{C} / L_{C}$ shall be introduced for the sake of comparison.

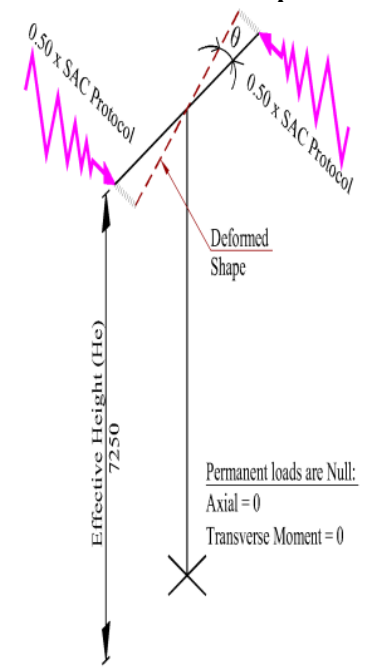

Figure 14: Analysis reference cases

Providing connection rotational stiffness as a relative values with respect to $\left(K_{T 0}\right)$ is a solid reference to evaluate the rigidity / flexibility of the studied connection under different loading conditions.

\section{Ultimate torsion}

By addressing relative ultimate torsion $\left(T_{U} / T_{U 0}\right)$ through Figure 15, it is clear that for both stiffened and un-stiffened plates the amount of torsion that can be sustained is very small when twisting action compared to bending in the longitudinal is low $(r=1)$, and this attributed to the fact that failure is controlled by flexural criterion, in the other hand when 
twisting actions superb the bending actions $r=3,5$; torsional failure becomes more probable and twisting defences of the connection are triggered, hence values of $\left(T_{U}\right)$ get higher.

It is clear that a remarkable jump in $\left(T_{U}\right)$ values take a place when torsion level promoted from $r=1$ to $r=3$, in spite of this; connection did not offer accountable increase in the ultimate torsion capacity when $r$ values increase from 3 to 5 , where the average increase is about $20 \%$, achieving its maxima for $95 \mathrm{~mm}$ thick base plate irrespective stiffening condition.

As per Figure 15a for un-stiffened base plate of $75 \mathrm{~mm}$ thickness effect of eccentricity level is almost neutral and only has presence at high torsion level $(r=5)$ where ultimate torsion reduced by $30 \%$ when concentric loads $(E / D=0)$ turned into eccentric loads $(E / D=$ $0.5,1.50)$. For the remaining studied cases; un-stiffened connection has not been affected by moving permanent loads from concentric application $(\mathrm{E} / \mathrm{D}=0)$, to eccentric application (E/D $=0.5)$, while a degradation in the $\left(T_{U}\right)$ up to $30 \%$ due to increase in the eccentricity to $\mathrm{E} / \mathrm{D}=$ 1.5. In contrary for stiffened connection (Figure $15 \mathrm{~b})$ a $30 \%$ reduction in $\left(T_{U}\right)$ occurs when $\mathrm{E} / \mathrm{D}$ increase from 0 to 0.5 , while extra increase in the eccentricity $(\mathrm{E} / \mathrm{D}=1.5)$ has not affect $\left(T_{U}\right)$. For $95 \mathrm{~mm}$ stiffened base plate effect of eccentricity on the maximum sustain torsion is negligible.

Effect of base plate thickness $\left(t_{P}\right)$ on the torsional capacity of the studied connection varies according to the applied action combination, for un-stiffened connection at concentric state, increasing base plate thickness $\left(t_{P}\right)$ is effective at " $r$ " values of 3 , and 5 , as the $\left(T_{U}\right)$ increases by $30 \%$ at $r=3$, when $\left(t_{P}\right)$ increases from $75 \mathrm{~mm}$ to $95 \mathrm{~mm}$, also at $r=5 ; 40 \%$ enhancement in torsional capacity achieved when $\left(t_{P}\right)$ increases from $55 \mathrm{~mm}$ to $75 \mathrm{~mm}$. At E/D $=0.50$ for un-stiffened configuration increasing base plate thickness from $55 \mathrm{~mm}$ to $75 \mathrm{~mm}$ is not effective, however further increase in base plate thickness to reach $95 \mathrm{~mm}$ has enhanced $\left(T_{U}\right)$ significantly by $50 \%$ in average. For high eccentricity level where $\mathrm{E} / \mathrm{D}=1.5$, presence of enhancing effect of $\left(t_{P}\right)$ appears only when base plate thickened from $55 \mathrm{~mm}$ to $75 \mathrm{~mm}$ where $\left(T_{U}\right)$ pumped up by $40 \%$, nevertheless thicker base plate is not advantageous for the connection in torsion resistance.
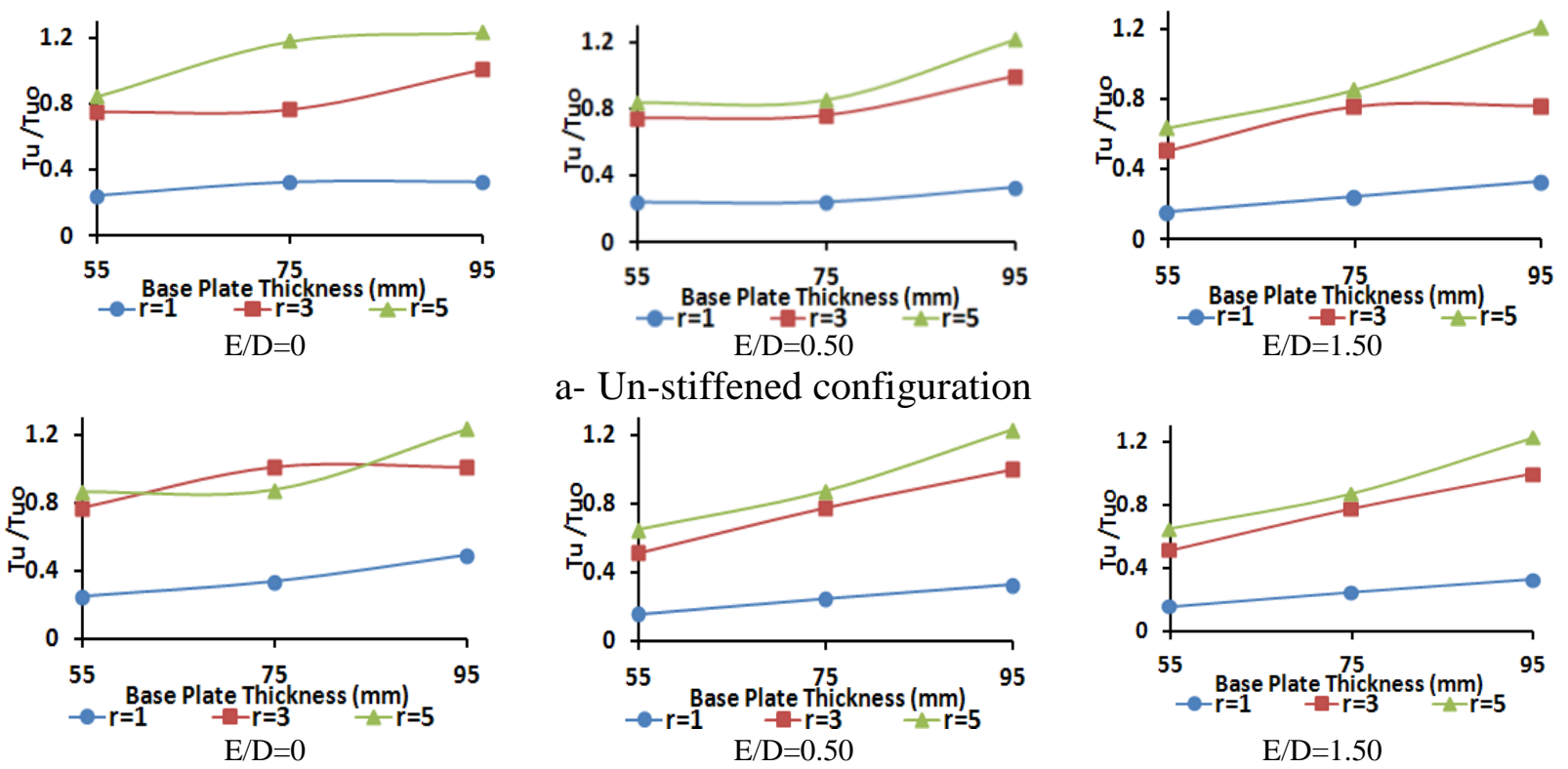

a- Un-stiffened configuration
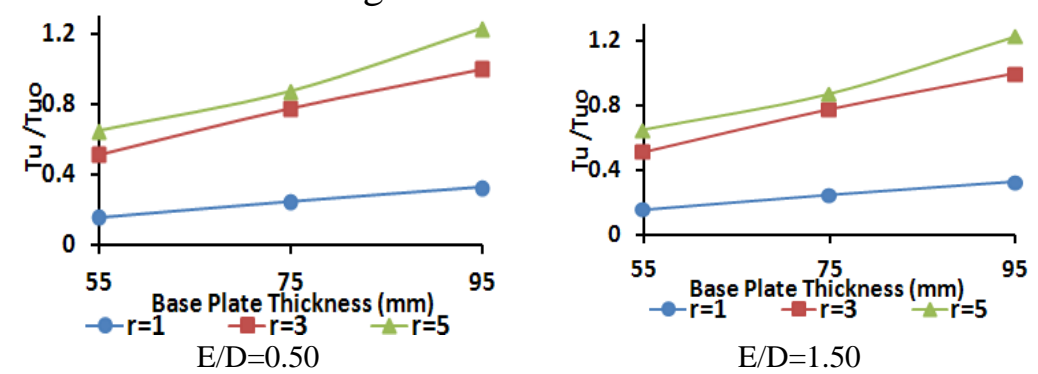

b- stiffened configuration

Figure 15: Relative Ultimate torsion variation with base plate thickness

For stiffened configuration subjected to eccentric loading a pattern for the effect of $\left(t_{P}\right)$ on $\left(T_{U}\right)$ has showed up, where increasing $\left(t_{P}\right)$ from $55 \mathrm{~mm}$ to $75 \mathrm{~mm}$ at low and medium torsion levels $(r=1,3)$ improves $\left(T_{U}\right)$ by $50 \%$ to $60 \%$; this gain in torsional capacity decreased almost to the half at severe torsion level $(r=5)$. Increasing base plate from $75 \mathrm{~mm}$ to $95 \mathrm{~mm}$ is also effective but with less efficiency, as the obtained gain in $\left(T_{U}\right)$ limited to $30 \%$ at $r=1,3$ while slightly increased at $r=5$.

For concentric loading $(\mathrm{E} / \mathrm{D}=0)$ the previously mentioned pattern is found at $r=1$, while at $r=3$ enhancing effect of increasing $\left(t_{P}\right)$ vanishes for plates thicker than $t_{P}=75 \mathrm{~mm}$, in the other hand at $r=5$ increasing base plate thickness feasible for $t_{P}>75 \mathrm{~mm}$. 
Comparing figures Figure 15a, Figure $15 \mathrm{~b}$ reveals that stiffening base connection almost did not exhibit improving effect on the torsional capacity.

\section{Ultimate twisting angle}

It is clear from Figure 16 where flexural failure is dominant $(r=1)$ the ultimate twisting angle $\left(\theta_{U}\right)$ is not affected by any variation in the connection configuration neither plate thickness nor stiffening condition, as well as gravity load state has minor effect on it.

From the aspect of torsional severity it is obvious that a remarkable jump in $\left(\theta_{U}\right)$ values take a place when torsion level promoted from $r=1$ to $r=3$, in spite of this; at base plate thickness $\left(t_{P}\right) 55 \mathrm{~mm}$, and $75 \mathrm{~mm}$ connection did not offer accountable increase in the ultimate torsion rotation when $r$ values increased from 3 to 5, however for the mentioned step up in " $r$ " values connection of $\left(t_{P}\right)$ of $95 \mathrm{~mm}$ thick showed an significant magnification for $\left(\theta_{U}\right)$ specially for un-stiffened configuration.

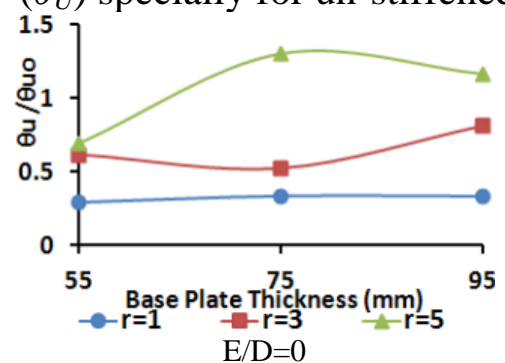

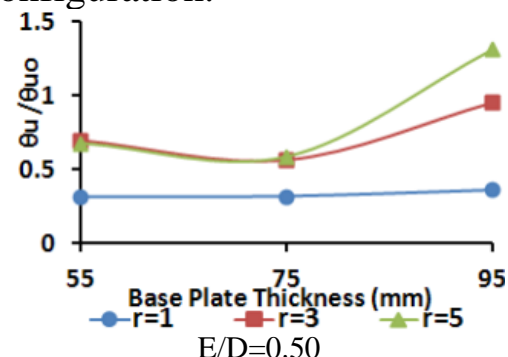

a- Un-stiffened configuration

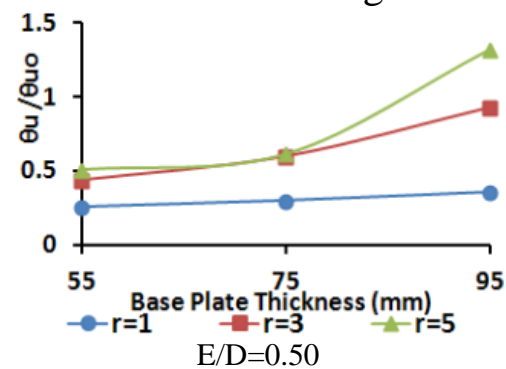

b- stiffened configuration
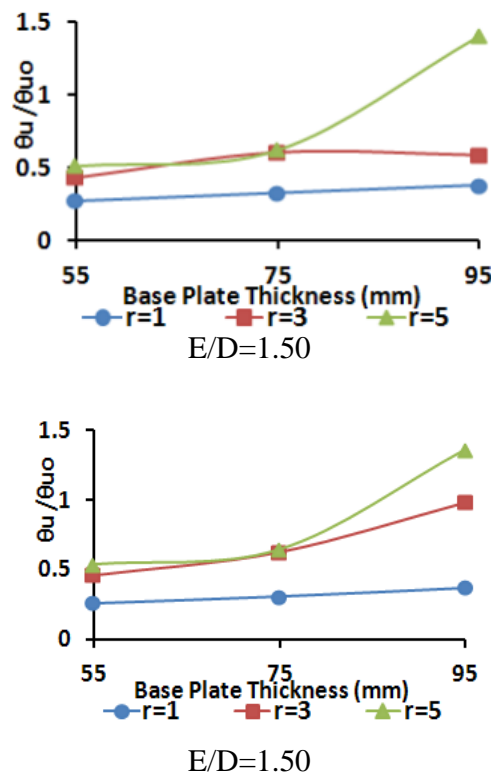

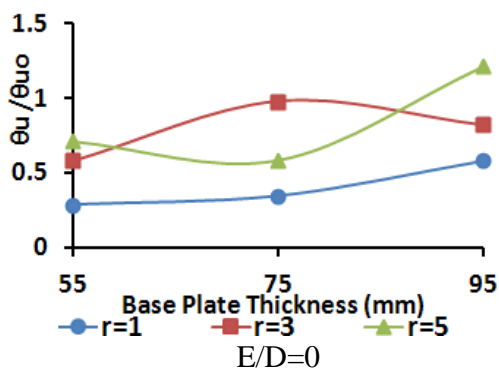

Figure 16: Relative ultimate twisting angle variation with base plate thickness

As extracted from Figure 16, in general eccentricity effect is minor on twisting deformation capacity of the connection especially for eccentric cases; however some exceptions are found as follows. For un-stiffened plate of $75 \mathrm{~mm}$ thick subjected to high torsion level $(r=5)$ the $\left(\theta_{U}\right)$ value dropped remarkably by $55 \%$ when changing loading from concentric combination to eccentric combination irrespective eccentricity level, this also witnessed for the stiffened configuration of the same base plate thickness at $r=3$. Finally for applied torsion conforming to " $r$ " value of 3; ultimate torsional angle recorded its maxima at $\mathrm{E} / \mathrm{D}=0.5$, and then a slight reduction in the $\left(\theta_{U}\right)$ took a place when E/D set to zero in the analysis, as well as $40 \%$ reduction in $\left(\theta_{U}\right)$ is exhibited when E/D increased to 1.50.

For connections subjected to low torsion stresses $(r=1)$; increasing base plate thickness slightly increases the expected $\left(\theta_{U}\right)$, this is also extended at $r=3,5$, however limited on eccentrically loaded connections with $t_{P}<75 \mathrm{~mm}$. In the other hand further increase of $\left(t_{P}\right)$ beyond $75 \mathrm{~mm}$ for connections subjected to eccentric permanent loads has a considerable reflection on the $\left(\theta_{U}\right)$ of these connections; as per Figure 16 at $r=3$ the ultimate twisting angle increased by $60 \%$ due to thickening base plate from $75 \mathrm{~mm}$ to $95 \mathrm{~mm}$, the mentioned increasing rate corresponding to the same thickening step has been doubled when torsion level upgraded to $r=5$. This pattern is also found for concentric loading, however unstiffened connection at $r=5$ and for stiffened connection at $r=3$ were off trend such that the previously monitored increase in the connection twisting tendency has showed up when $\left(t_{P}\right)$ increased from $55 \mathrm{~mm}$ to $75 \mathrm{~mm}$, while introducing thicker plate than $75 \mathrm{~mm}$ has slightly decreased the exhibited $\left(\theta_{U}\right)$ values.

The pair figures Figure 16a, Figure 16b illustrate together the impact of adding stiffener to the connection assembly in absorbing extra twisting rotation, and as generally 
concluded from these figures stiffeners has no sensible impact on the $\left(\theta_{U}\right)$, while in detailed level perspective, a two opposite events occurred when stiffening $75 \mathrm{~mm}$ base plate subjected to concentric permanent loads $(\mathrm{E} / \mathrm{D}=0)$, first is increasing ultimate twisting angle by $30 \%$ at " $r$ " value equals to 3 , second is decreasing ultimate twisting angle by almost $30 \%$ when " $r$ " increased to 5. At $r=3$ for eccentrically loaded connection with $\mathrm{E} / \mathrm{D}=1.5$ the $95 \mathrm{~mm}$ base plate tolerated for an extra torsional rotation by adding stiffener.

\section{Torsoinal stiffness}

Previous sections demonstrated the torsional properties in terms of strength and deformation at the ultimate state of base connection with respect to the proposed study parameters; this section shall discuss the elastic features of the studied connections in term of torsional stiffness.

Figure 17 represents the envelope curve of hysteretic torsional response for the studied connection for cyclic, the figure is divided into 3 ranges; first range is "trigger range" where the connection shows a soft response to the applied torsion, this range ends at what so called "trigger point" to start the second range "elastic range" at which the torsional defenses of the connections are triggered and the real elastic stiffness $\left(K_{T}\right)$ controls the connection behavior, this stage reaches its end at the yield state of the connection succeeded by the final stage "post elastic", that featured by sharp reduction in the connection stiffness at yield point terminated by connection failure.

Figure 18 represents the bolt deformation for two successive drift ratios at early stage of the applied cyclic loading (SAC protocol); as shown in the figure the bolt exhibited large lateral deformation at the initial drift ratio $(0.375 \%)$ due connection slip resulting from gap closing between bolt hole and the bolt itself, however at the next level of drift $(0.50 \%)$ slippage brought bolts into bearing and the provided deformation of the bolt became inconsiderable compared to the recorded deformation at the initial drift ratio. This clarifies the initial soft stiffness of the connection and how its stiffness triggered after slippage as shown in the elastic range (Figure 17).

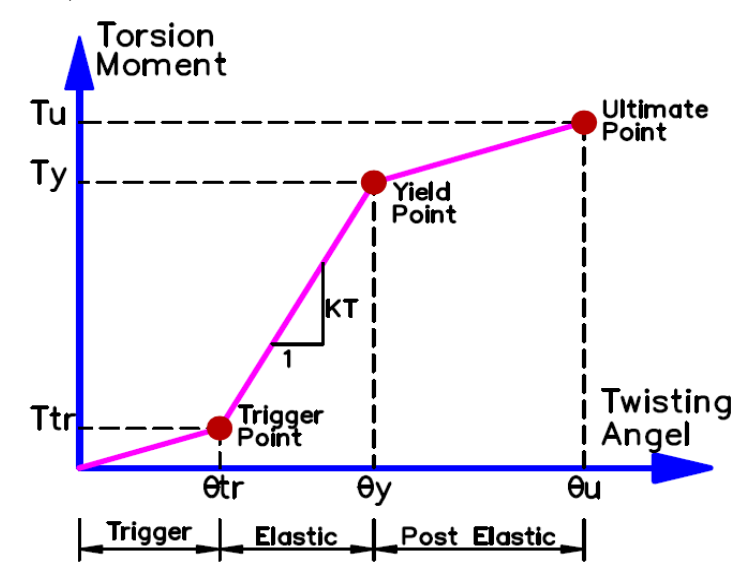

Figure 17: Typical torsion - twisting angle envelope curve of the hysteretic cyclic torsion response

As per Figure 19 the torsional stiffness $\left(K_{T}\right)$ at its lowest when flexural stresses are dominant $(r$ $=1)$, also Figure 19 provides two trends for the variation of $\left(K_{T}\right)$ with respect to the torsion level represented in " $r$ "; first trend is monitored for un-stiffened connection showing a minor effect on the torsional stiffness due changing torsion level for base plate thickness $55 \mathrm{~mm}$, and $95 \mathrm{~mm}$, while for base plate thickness of $75 \mathrm{~mm}$ this trend is achieved only for connection subject to big eccentric gravity loading $(\mathrm{E} / \mathrm{D}=1.5)$ at higher torsional level $(r=3, r=5)$, however for the same base plate thickness, increasing " $r$ " from 1,3 increased $\left(K_{T}\right)$ by $50 \%$.Second trend is monitored for stiffened connection where " $r$ " has an effect on the connection tendency to twist with variable severity level; when tracing the previously mentioned trend it was found that increasing " $r$ " from 1, to 3 enhanced $\left(K_{T}\right)$ values by $20 \%$ in average at concentric loading state $(\mathrm{E} / \mathrm{D}=0)$, and $40 \%$ at $\mathrm{E} / \mathrm{D}=0.5,1.5$ for $75 \mathrm{~mm}$ stiffened base plate, however this enhancement is lost for further increase in " $r$ " at $\mathrm{E} / \mathrm{D}=0,0.50$, while for big eccentricity level $(\mathrm{E} / \mathrm{D}=1.50)$ increasing " $r$ " from 3 to 5 maintained the value for torsional stiffness. 


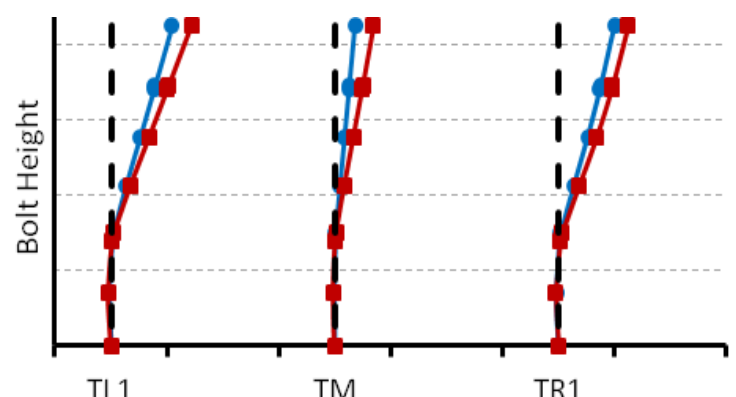

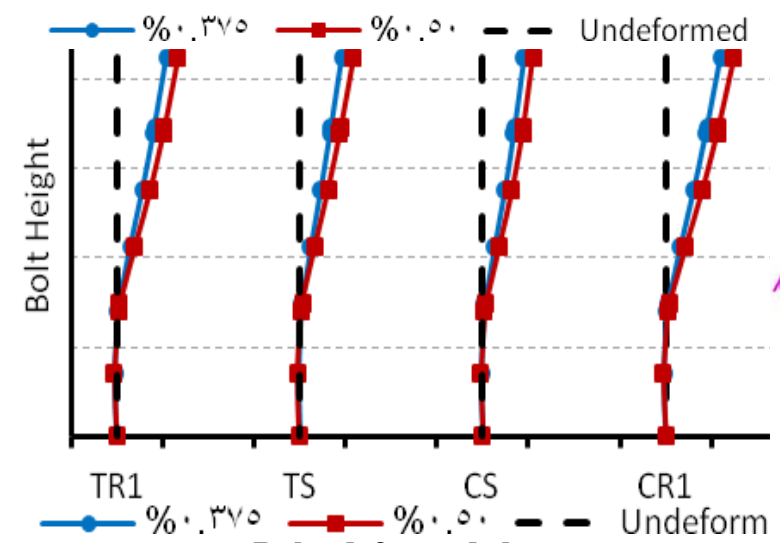

a- Bolts deformed shape

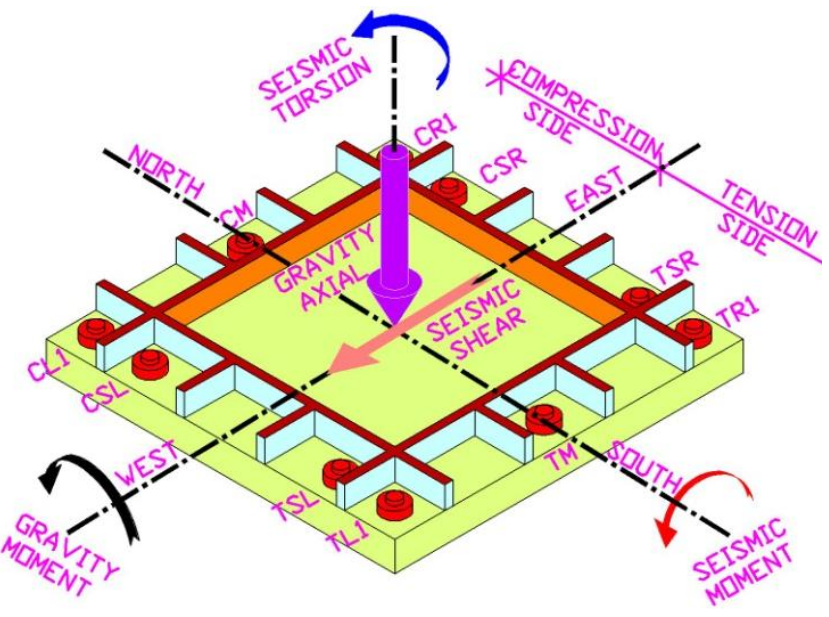

b- Base connection actions and bolts ID

Figure 18: Bolt deformed shape for early stage of cyclic loading

Further analysis for Figure 19 tells that the effect of base plate thickness on the $\left(K_{T}\right)$ only matters for connections severely stresses by permanent loads $(\mathrm{E} / \mathrm{D}=1.50)$ and seismic torsion $(r=3,5)$ where increasing base plate thickness from $55 \mathrm{~mm}$ to $75 \mathrm{~mm}$ raised the $\left(K_{T}\right)$ by $40 \%$, and $60 \%$ for $r=3$, and $r=5$ respectively, however further reduction to $95 \mathrm{~mm}$ reduces the $\left(K_{T}\right)$ by $20 \%$ lesser than values obtained at the $75 \mathrm{~mm}$ base plate thickness. For the remaining studied cases base plate thickness is not a present factor in the torsional stiffness. As per Figure 19, neither eccentricity level (E/D) nor does stiffening condition have a noticeable effect on the torsional stiffness of the studied connections.

As a general perspective it can be said that torsional stiffness for studied connection achieved low resistance for twisting rotation, and relaying on it as rigid fixed model is questionable. Figure 19 emphasizes this fact where studied connection achieved an average of $65 \%$ of the fixed base model $\left(K_{T 0}\right)$, However base plate of $75 \mathrm{~mm}$ thickness offered a high twisting resistance recording $85 \%$ of $\left(K_{T O}\right)$, which attributed to the combined failure mode that exhibited yielding in the bolts and base plate as well. stiffened connection concentrically loaded; is introduced, that almost simulates the rigid fixed base, while for eccentrically loaded stiffened assembly the connection showed more tendency for rotation that provided connection stiffness hardly reached $55 \%$ of $\left(K_{F 0}\right)$.
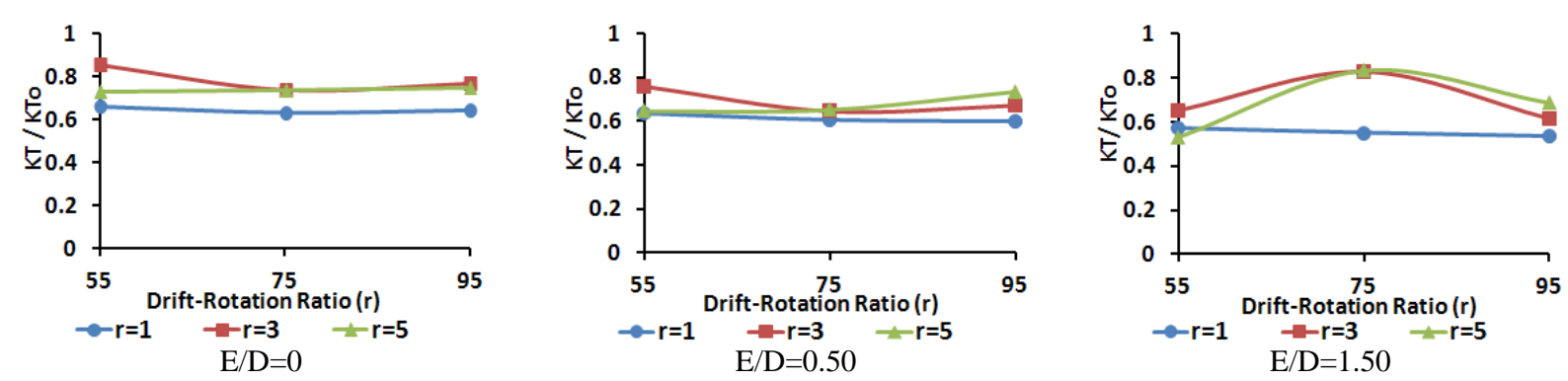

\section{a- Un-stiffened configuration}



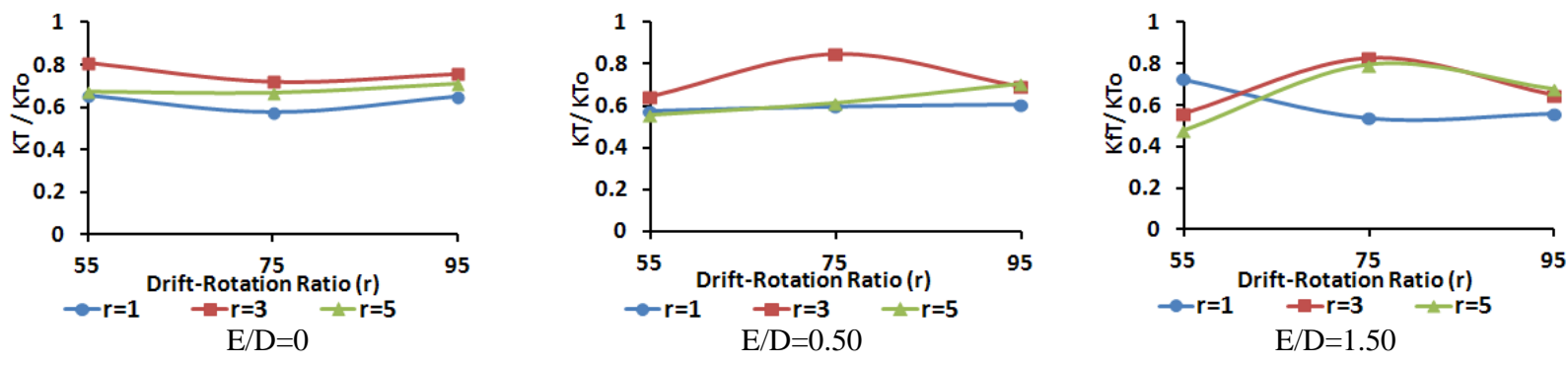

Figure 19: Typical Relative torsional stiffness variation with base plate thickness

\section{Conclusions}

The Following Conclusions Can Be Drawn From The Analytical Study:

1-Sustainable Torsion Terms $\left(T_{u}, \Theta_{u}\right)$ Are Inversely Proportioned To The Seismic Flexural To Torsion Ratio, However This Ratio Only Matters For The Connection Torsional Stiffness $\left(K_{t}\right)$ Of Stiffened Configuration.

2- $\left(T_{u}\right)$ Of The Stiffened Configuration Is Mainly Affected By The Nature Of Gravity Load Whether It Is Concentric Or Eccentric, While What Counts In The Ultimate Torsion Capacity Of Un-Stiffened Configuration Is The Intensity Of Gravity Moment, Furthermore $\left(\Theta_{u}\right)$ Is Not Affected By The Neither The Nature Nor The Eccentricity Of Gravity Loads.

3-Base Plate Thickening Effectively Increases $\left(T_{u}\right)$, With More Feasibility In The UnStiffened Assembly, However $\left(T_{p}\right)$ Has A Limited Effect On The Twisting Deformation $\left(\Theta_{u}\right)$, And Torsional Stiffness $\left(K_{t}\right)$, Hence A Great Increase In Base Plate Thickness Is Required To Absorb Extra Torsional Demands At Failure.

4-Stiffening Condition Of Base Connection Has A Limited Presence In The Expected Value Of $\left(T_{u}\right)$ And Unnoticeable Impact On $\left(\Theta_{u}\right)$, And $\left(K_{t}\right)$.

5- $\left(K_{t}\right)$ For Studied Connection Is, Hence Rigid Fixed Model Is Questionable. However Base Plate Of $75 \mathrm{~mm}$ Thickness Offered The Highest Twisting Resistance Recording 85\% Of $\left(K_{t 0}\right)$, Which Attributed To The Combined Failure Mode That Exhibited Yielding In The Bolts And Base Plate As Well.

\section{Acknowledgment}

The Author Gratefully To First And Foremost Thank God For His Giving's And Guidance, Also A Thankfully Acknowledgement Goes To Professor Dr. Sherif Safar For His Endless Support. I Express My Gratitude To Mr. Sayed Ibrahim, Mr. Ibrahim Abdelhaleem And To Dr. Mohamed Helal For Their Continuous Encouragement. Last But Not Least To My Wife Who Is Always There For Me.

\section{REFERENCES}

[1] M. Fahmy, "Seismic Behavior Of Moment - Resisting Steel Column Bases," Doctor Of Philosophy (Civil Engineering) The University Of Michigan, Michigan, 1999.

[2] P. Tirasit, K. Kawashima And G. Watanabe, "Effect Of Combined Cyclic Flexural Torsional Loading On The Seismic Performance Of Rc Columns," In Earthquakes Engineering Research Institute, 100th Anniversary Earthquakes Conference, San Francisco, Califorenia, 2006.

[3] P. Clark, K. Frank, H. Krawinkler And R. Shaw, "Protocol For Fabrication, Inspection, Testing, And Documentation Of Beam-Column Connection Tests And Other Expremental Specimens," Sac Joint Venture, Report No. Sac/Bd-97/02, Sacramento, California, 1997.

[4] T. C. A., H. T.C. And G. P.R., "Cyclic Behavior Of Structural Base Plate Connnection With Fastening Failure: Component Test Results," In Tenth U.S. National Conference On Earthquake Engineering, Frontiers Of Earthquake Engineering, Alaska, 2014.

[5] B. J., M. G.A., C. J.G. And C. C.G., "Experimental Studies On Cyclic Behaviour Of Steel Base Plate Connections Considering Anchor Bolts Post Tensioning," In New Zealand Society For Earthquake Engineering Conference, Christchurch, New Zealand, 2014. 
[6] C. Yao, K. Shoichi And Y. Satoshi, "Hysteretic Behavior Of Exposed Column Bases In Buckling Restrained Braced Frames," In Fifthteenth World Conference On Earthquakes Engineering, Lisbon, 2012.

[7] S. E. Abdel Raheem And T. Hayashikawa, "Seismic Performance Of Semi-Rigid Base Connection Model Of Cable-Stayed Bridge Tower," International Jouranl Of Civil And Structural Engineering, Vol. 3, No. 2, 2012.

[8] D.-Y. Lee, S. C. Goel And B. Stoj, "Exposed Column-Base Plate Connections Bending About Weak Axis: I. Numerical Parametric Study," International Journal Of Steel Structures, 2008.

[9] M. F. James And A. K. Lawrence, "Base Plate And Anchor Rod Design," Steel Design Guide 1, Aisc, 2006.

[10] A. N. Jaswant And M. C.V.R., "P-V-M Interaction Curves For Seismic Design Of Steel Column Base Connections," American Institute Of Steel Construction, Engineering Journal, Third Quarter, 2002.

[11] D.-Y. Lee, S. C. Goel And B. Stoj, "Exposed Column-Base Plate Connections Bending About Weak Axis: Ii Expremintal Study," International Journal Of Steel Structures, 2008.

[12] "Eurocode 3.: Design Of Steel Structures, Part 1 - 8: Design Of Joints," Bs En 1993-1-8, 2005. 